\title{
Quercetin prevents necroptosis of oligodendrocytes by inhibiting macrophages/microglia polarization to M1 phenotype after spinal cord injury in rats
}

Hong Fan ${ }^{1,2+}$, Hai-Bin Tang ${ }^{3+}$, Le-Qun Shan ${ }^{1}$, Shi-Chang Liu', Da-Geng Huang ${ }^{1}$, Xun Chen ${ }^{4}$, Zhe Chen ${ }^{1}$, Ming Yang ${ }^{1}$, Xin-Hua Yin ${ }^{1}$, Hao Yang ${ }^{1 *}$ and Ding-Jun Hao ${ }^{1 *}$

\begin{abstract}
Background: Oligodendrocytes (OLs) death after spinal cord injury (SCI) contributes to demyelination, even leading to a permanent neurological deficit. Besides apoptosis, our previous study demonstrated that OLs underwent receptor-interacting serine-threonine kinase 3(RIP3)/mixed lineage kinase domain-like protein (MLKL)-mediated necroptosis. Considering that necroptosis is always accompanied with pro-inflammatory response and quercetin has long been used as anti-inflammatory agent, in the present study we investigated whether quercetin could inhibit necroptosis of OLs and suppress the M1 macrophages/microglia-mediated immune response after SCl as well as the possible mechanism.
\end{abstract}

Methods: In this study, we applied quercetin, an important flavonoid component of various herbs, to treat rats with $\mathrm{SCl}$ and rats injected with saline were employed as the control group. Locomotor functional recovery was evaluated using Basso-Beattie-Bresnahan (BBB) scoring and rump-height Index (RHI) assay. In vivo, the necroptosis, apoptosis, and regeneration of OLs were detected by immunohistochemistry, 5'-bromo-2'-deoxyuridine (BrdU) incorporation. The loss of myelin and axons after SCl were evaluated by Luxol fast blue (LFB) staining, immunohistochemistry, and electron microscopic study. The polarization of macrophages/microglia after $\mathrm{SCl}$ and the underlying mechanisms were detected by quantitative reverse transcription-polymerase chain reaction (qRT-PCR) and immunohistochemistry. In vitro, the ATP and reactive oxygen species (ROS) level examination, propidium iodide (PI) labeling, and Western blotting were used to analyze the necroptosis of cultured OLs, while the signaling pathways-mediated polarization of cultured macrophages/microglia was detected by qRT-PCR and Western blotting.

Results: We demonstrated that quercetin treatment improved functional recovery in rats after SCl. We then found that quercetin significantly reduced necroptosis of OLs after SCI without influencing apoptosis and regeneration of OLS. Meanwhile, myelin loss and axon loss were also significantly reduced in quercetin-treated rats, as compared to $\mathrm{SCl}+$ saline control. Further, we revealed that quercetin could suppress macrophages/microglia polarized to M1 phenotype through inhibition of STAT1 and NF-KB pathway in vivo and in vitro, which contributes to the decreased necroptosis of OLs.

Conclusions: Quercetin treatment alleviated necroptosis of OLs partially by inhibiting M1 macrophages/microglia polarization after SCl. Our findings suggest that necroptosis of OLs may be a potential therapeutic target for clinical SCl.

Keywords: Spinal cord injury, Oligodendrocyte, Necroptosis, Macrophages/microglia, Quercetin

\footnotetext{
*Correspondence: yanghao.71_99@yahoo.com; haodingjun@126.com

${ }^{+}$Hong Fan and Hai-Bin Tang contributed equally to this work.

${ }^{1}$ Shaanxi Spine Medicine Research Center, Translational Medicine Center,

Department of Spine Surgery, Hong Hui Hospital, Xi'an Jiaotong University,

555 You Yi Dong Road, Xi'an 710054, Shaanxi, China

Full list of author information is available at the end of the article
}

(c) The Author(s). 2019 Open Access This article is distributed under the terms of the Creative Commons Attribution 4.0 International License (http://creativecommons.org/licenses/by/4.0/), which permits unrestricted use, distribution, and reproduction in any medium, provided you give appropriate credit to the original author(s) and the source, provide a link to the Creative Commons license, and indicate if changes were made. The Creative Commons Public Domain Dedication waiver (http://creativecommons.org/publicdomain/zero/1.0/) applies to the data made available in this article, unless otherwise stated. 


\section{Background}

The secondary injury after spinal cord injury (SCI), more tightly related to severity of neurological deficit, includes cell death, demyelination, axonal degeneration, and inflammatory response [1-3]. Among these, oligodendrocytes (OLs) death after SCI contributes to demyelination of spared axons, even leading to a permanent neurological deficit [4].

Given that the survival of OLs is crucial for functional recovery after $\mathrm{SCI}$, in the present study we focused on death and regeneration of OLs. Apoptosis of OLs occurs mainly in the first several hours after SCI, and the injury is accompanied by chronic inflammatory demyelination [5-7], indicating other types of cell death accounted for the delayed loss of OLs. It has been reported that necroptosis of OLs mediates axonal degeneration in ALS [8]. Our previous study demonstrated that some OLs were receptor-interacting serine-threonine kinase 3(RIP3)/mixed lineage kinase domain-like protein (MLKL)-positive after SCI, indicating that necroptosis occurred in OLs [9]. The chronic, detrimental, M1 macrophages/microglia-mediated inflammatory response contributed to necroptosis of astrocytes after SCI $[10,11]$. Likewise, the dominance of M1 sub-population might play a role in necroptosis of OLs. Thus, there is an urgent need for new treatment strategies to prevent OLs death and to ameliorate the M1 macrophages/microglia-mediated immune response after $\mathrm{SCI}$.

Quercetin, an important flavonoid component of various herbs, has long been used as antioxidant and anti-inflammatory agent in traditional Chinese medicine [12-14]. It was confirmed to have neuroprotective effects on SCI by reducing death of neurons, decreasing neural tissue damage, and inhibiting inflammatory responses [15-18]. However, the effects of quercetin on survival of OLs and polarization of macrophages/microglia after SCI still remain unclear.

In this study, we investigated whether quercetin promoted OLs survival and suppressed the detrimental M1type immune response after $\mathrm{SCI}$, and explored the possible mechanism. We first showed that quercetin improved functional recovery after SCI. We then demonstrated that quercetin significantly reduced necroptosis of OLs after SCI without affecting apoptosis and regeneration of OLs. Meanwhile, quercetin significantly reduced myelin and axon loss after SCI. We further revealed that quercetin suppressed macrophages/ microglia polarized to $\mathrm{M} 1$ phenotype through inhibition of STAT1 and NF-kB pathway, which contributes to the ameliorated necroptosis of OLs. Taken together, our study demonstrated that quercetin alleviated necroptosis of OLs in part by suppressing M1 macrophages/microglia polarization through inhibition of STAT1 and NFкB pathway after SCI.

\section{Methods}

\section{Experimental design}

Male Sprague-Dawley rats (200-250 g) were purchased from the Laboratory Animal Center of Xi'an Jiaotong University. This study was carried out in accordance with the recommendations of 'Animal Care and Use, Committee of Xi'an Jiaotong University.' The protocol was approved by the 'Committee of Xi'an Jiaotong University.' A total of 120 rats were equally randomized into three groups: Sham group, SCI + saline group, and SCI + quercetin group. The sham group received only laminectomy, and the vehicle $(1 \mathrm{ml}$ sterile saline plus $1 \%$ DMSO) was administrated via intraperitoneal injection (i.p.) after SCI as SCI + saline group. According to the previous finding that functional recovery correlates treatment duration of quercetin after SCI [19], $7.5 \mathrm{mg} /$ $\mathrm{kg}$ quercetin (Sigma-Aldrich, St. Louis, MO, USA) in $1 \mathrm{ml}$ vehicle was administered i.p. twice daily for 10 days after SCI.

\section{Animal model of spinal cord injury}

Rats were anesthetized with $1 \%$ sodium pentobarbital $(60 \mathrm{mg} / \mathrm{kg})$, and bilateral laminectomy of vertebrae T8 was performed to expose the spinal cord. The spinal cord crush model was made by a mechanical device [20]. Briefly, we used the forceps $(53327 \mathrm{~T}, 66$ Vision-Tech Co., Ltd., China) mounted onto the device to clamp the cord, with the forceps tips of $0.5-\mathrm{mm}$ width when fully closed, and the duration of the injury remained for $20 \mathrm{~s}$. After crush, manual bladder expression was performed once a day, until reestablishment of micturition reflex. Depending on the following experiments, rats were sacrificed at 10 and 21 days post injury (dpi).

\section{Behavioral tests \\ Basso-Beattie-Bresnahan scores}

The Basso-Beattie-Bresnahan (BBB) scores were used to test rat open-field locomotion at 1 day before, and 1,3 , $7,10,14$, and 21 days after spinal cord injury (SCI) following methodology of BBB [21]. Functional scores ranges from 0 to 21 points in terms of the ankle, hip, knee, and trunk movement, as well as the coordination of each rat. The locomotion scores was observed and recorded by two independent observers blinded to the experiments.

\section{Rump-height Index assay}

The rats were video-recorded during walking through the left to right side on a runway bar $(160 \mathrm{~cm}$ long, $10 \mathrm{~cm}$ wide, and $8 \mathrm{~cm}$ thick) at 1 day prior to and 1,3 , $7,10,14$, and 21 days post-SCI. The rump-height Index (RHI) is defined as the height of the rump, normalized to the thickness of the beam measured along the same vertical line [22]. To minimize the variations of pre- 
surgery RHI of each animal, the standardized RHI (dividing post-injury value by pre-injury value) was applied for comparisons.

\section{Tissue processing}

Seven days after SCI or at the end of the 21-day observation period, rats were anesthetized and trans-cardially perfused with $200 \mathrm{ml}$ of saline, followed by $400 \mathrm{ml}$ of $4 \%$ PFA. Subsequently, 2-cm spinal cord segments encompassing the lesion site were obtained and cryoprotected in sucrose (30\% in $0.1 \mathrm{M}$ phosphate buffer, $\mathrm{pH}$ 7.4) until the blocks sank.

Both serial transverse sections (12- $\mu \mathrm{m}$-thick) and serial sagittal sections $(12-\mu \mathrm{m}$-thick) were cut on a cryostat and prepared for immunostaining.

\section{Immunohistochemistry}

After blocking in $0.01 \mathrm{M}$ PBS containing 3\% BSA and $0.3 \%$ Triton X-100 for $30 \mathrm{~min}$, sections were incubated with primary antibodies in humidified boxes overnight at $4{ }^{\circ} \mathrm{C}$. The primary antibodies used in the experiment included rabbit anti-RIP3 (1:500, ENZO, New York, NY, USA), rat anti-MLKL (1:200, Millipore, Billerica, MA, USA), rabbit anti-phosphorylated MLKL (pMLKL) (1: 300, Abcam, Cambridge, Cambridgeshire, UK), mouse anti-CC1 (1:600, Milipore, Billerica, MA, USA), rabbit anti-iNOS (1:200, Abcam, Cambridge, Cambridgeshire, UK), goat anti-Arginase1(1:200, Santa Cruz Biotechnology, Dallas, Texas, USA), mouse anti-glial fibrillary acidic protein (GFAP,1:2000, Sigma-Aldrich, St. Louis, MO, USA), rabbit anti-MBP(1:200, Abcam, Cambridge, Cambridgeshire, UK), mouse anti-NF200 (1:200,Abcam, Cambridge, Cambridgeshire, UK), rabbit anti-Cleaved Caspase-3(1:200, Cell Signaling, Danvers, MA, USA), goat anti-Iba-1(1:200,Abcam, Cambridge, Cambridgeshire, UK), mouse anti-PDGFR $\alpha$ (1:200, Cell Signaling, Danvers, MA, USA), rat anti-BrdU (1:300,Abcam, Cambridge, Cambridgeshire, UK), and rabbit anti-Olig2 (1: 200, Abcam, Cambridge, Cambridgeshire, UK). The sections were then incubated with the corresponding secondary antibodies conjugated with Alexa Fluor 594 (donkey anti-rabbit IgG or donkey anti-mouse IgG, 1: 1000, Jackson ImmunoResearch, West Grove, PA, USA), Alexa Fluor 488 (donkey anti-rat IgG or donkey antimouse IgG, 1:1000, Jackson mmunoResearch, West Grove, PA, USA) in a dark environment for at RT $1 \mathrm{~h}$. The sections were counterstained with Hoechst 33342 to identify all nuclei. Sections were examined and photographed under a confocal microscope (LSM 800, Zeiss, Oberkochen, Germany).

\section{BrdU incorporation and detection}

For detection of regeneration of OLs, BrdU $(100 \mathrm{mg} / \mathrm{kg}$, Sigma, St. Louis, MO, USA) injection was initiated at
$24 \mathrm{~h}$ after injury, once a day for 9 days. Rats were sacrificed at 10 days after injury. $\mathrm{HCl}$ treatment $(2 \mathrm{~N} \mathrm{HCl}$, $30 \mathrm{~min}$ at $37^{\circ} \mathrm{C}$ ) was performed for BrdU staining.

\section{Luxol fast blue staining}

For Luxol fast blue (LFB) staining, serial transverse cryosections $(12 \mu \mathrm{m}$ thickness) were stained as previously described. Briefly, slides were incubated in $0.1 \%$ LFB (Sigma, St. Louis, MO, USA) in acidified 95\% ethanol overnight at $60{ }^{\circ} \mathrm{C}$. The slides were then differentiated and counterstained with $0.05 \%$ lithium carbonate and cresyl violet solution. LFB-stained sections at $2000 \mu \mathrm{m}$ rostral and caudal to the injury site, as well as at the lesion epicenter, were analyzed for myelin sparing. The spared myelin in white matter was quantified by Image pro-plus (Media Cybernetics, Silver Spring, USA).

\section{Electron microscopic study}

Rats were deeply anesthetized with $1 \%$ sodium pentobarbital intraperitoneally $(60 \mathrm{mg} / \mathrm{kg}$ body weight) and perfused with $200 \mathrm{ml}$ saline, followed by $500 \mathrm{ml} 4 \%$ PFA containing $0.05 \%$ glutaraldehyde. Then the injured cord was removed and postfixed in the same fixative solution for $4 \mathrm{~h}$ at $4{ }^{\circ} \mathrm{C}$. Serial coronal sections of $50 \mu \mathrm{m}$ thickness were prepared with a vibratome (VT 1000S, Leica, Wetzlar, Hesse, Germany), then placed in 0.01 M PBS containing $25 \%$ sucrose and $10 \%$ glycerol for $2 \mathrm{~h}$ for cryoprotection. The sections were then immersed in $0.5 \%$ osmium tetroxide for $1 \mathrm{~h}$, dehydrated with graded ethanol series, then in propylene oxide, and finally flatembedded in Epon812 (SPI-CHEM). After trimming under a stereomicroscope, the sections were glued onto blank resin stubs. Serial ultrathin sections were cut with an ultramicrotome (Leica EM UC6, Leica, Wetzlar, Hesse, Germany) and mounted on mesh grids. They were then counterstained with uranyl acetate and lead citrate, observed, and photographed under an electron microscope (JEM-1230, JEOL Ltd., Akishima, Tokyo, Japan) equipped with CCD camera and its application software (832 SC1000, Gatan, Pleasanton, CA, USA).

\section{Axonal diameter and $\mathrm{G}$ ratio determination}

Axonal diameter was determined from counting of about 200 axons in white matter of the injured segments of three rats at $21 \mathrm{dpi}$ using Image J software. G-ratio was determined as the ratio of inner axonal diameter to the total outer diameter of each axon fiber of about the same 200 axons of three rats at $21 \mathrm{dpi}$.

\section{Primary microglial culture and polarization}

Microglia were isolated from P2 Sprague-Dawley rat pups as described. Briefly, the spinal cord was dissected, mechanically dissociated, and then digested with $0.125 \%$ trypsin/0.02\% EDTA. Cells were plated onto poly-D- 
lysine-coated culture flasks and cultured in DMEM (Gibco, Waltham, USA) containing $10 \%$ FCS at $37{ }^{\circ} \mathrm{C}$ with $5 \% \mathrm{CO}_{2}$. The medium was exchanged every 3 days and at about 8th day when cells became confluent, the flasks were shaken with a shaker at $260 \mathrm{rpm}$ for $0.5 \mathrm{~h}$ to obtain microglia. The quality of microglia was analyzed by immunostaining of Iba1 (>90\% Iba1 $\left.{ }^{+}\right)$.

After about 7 days culture, microglial cells were treated with LPS (100 ng/ml, Sigma, Santa Clara, USA) and IFN- $\gamma$ (20 ng/ml, Peprotech, Rocky Hill, USA) for M1 polarization, or quercetin plus LPS and IFN- $\gamma$, followed by medium replacement. Twenty-four hours later, the conditioned medium (M1 CM and Q-M1 CM) was obtained.

\section{Primary oligodendrocytes culture}

After microglia was harvested, with another overnight shaking at $280 \mathrm{rpm}$, cell suspension was harvested and seeded on tissue culture dishes (Corning, NY, USA) for $40 \mathrm{~min}$. The dishes were then gently swirled every $6 \mathrm{~min}$ to remove contaminating astrocytes and microglia. The non-adherent cells were seeded onto poly-D-lysinecoated culture dishes in DMEM containing 10\% FCS at density of $5 \times 10^{5}$ cells $/ \mathrm{cm}^{2}$ for $3 \mathrm{~h}$, and the medium was then changed by oligodendrocyte precursor cells (OPCs) proliferation medium (chemically defined medium with $10 \mathrm{ng} / \mathrm{ml}$ PDGF-AA and $10 \mathrm{ng} / \mathrm{ml} \mathrm{bFGF}$ ). Three days later, OPCs were isolated with DMEM/F12 containing $0.01 \%$ EDTA, $0.2 \mathrm{mg} / \mathrm{ml}$ DNase I, and $5 \mathrm{mg} / \mathrm{ml}$ insulin, and seeded in differentiation medium (chemically defined medium with $30 \mathrm{nM}$ triiodothyronine and $5 \mathrm{mg} /$ $\mathrm{ml} \mathrm{N}$-acetyl-L-cysteine). Mature oligodendrocytes (OLs) were obtained 5 days later. Chemically defined medium contains high glucose DMEM/F12 supplemented with $4 \mathrm{mM}$ L-glutamine, $50 \mathrm{mg} / \mathrm{ml}$ transferrin, $1 \mathrm{mM}$ sodium pyruvate, $5 \mathrm{mg} / \mathrm{ml}$ insulin, and $10 \mathrm{ng} / \mathrm{ml} \mathrm{D-biotin.} \mathrm{The}$ mature OLs were identified by MBP staining.

\section{Western blotting}

About $1.5 \mathrm{~cm}$ length of injured cord segments or primary cultured microglia were harvested and homogenized with ice-cold radio-immunoprecipitation assay (RIPA) lysis buffer. Proteins were separated in 10\% SDS-PAGE gel, and transferred onto polyvinylidene difluoride (PVDF) membrane. The membranes were first blocked with $5 \%$ non-fat milk in TBST for $1 \mathrm{~h}$ at RT, and incubated with primary antibodies for RIP3 (1:800, ENZO, New York, NY, USA), MLKL (1:500, Millipore, Billerica, MA, USA), pMLKL (1:500, Abcam, Cambridge, Cambridgeshire, UK), iNOS(1:500, Abcam, Cambridge, Cambridgeshire, UK), p-STAT1 (1:1000, Cell Signaling, Danvers, MA, USA), nuclear factor-kappa B (NF-kB) (P65,1:1000, Cell Signaling, Danvers, MA, USA), phospho-NF-kB p65/p-P65 (1:1000, Cell Signaling,
Danvers, MA, USA), or beta-actin (1:5000, Sigma, Santa Clara, USA) at $4{ }^{\circ} \mathrm{C}$ overnight. The membranes were then incubated with corresponding horseradish peroxidaseconjugated secondary antibodies (1:5000, Jackson ImmunoResearch, West Grove, PA, USA) at RT for $1 \mathrm{~h}$. The immunoreactive bands were scanned with the Bio-Rad Image Lab system. The expression level was measured by ImageJ software (NIH, USA) corresponding to the band intensities.

\section{Quantitative reverse transcription polymerase chain reaction}

Total RNA was extracted from $1.5 \mathrm{~cm}$ length of injured cord segments or microglia using TRizol (Invitrogen, Carlsbad, CA, USA) following the manufacturer's instructions. Complementary DNA synthesis was performed using a Reverse Transcriptase kit (Invitrogen, Carlsbad, CA, USA). Real-time PCR for TNF $\alpha$, iNOS, CD86, CD206, Arginase1, IL-4, IL-10, IL-12, IL-1 $\beta$, and TGF $\beta$ were performed with SYBR Green (TaKaRa, Dalian, China) using Bio-Rad 96 Real-time PCR system (Bio-Rad Laboratories, Hercules, CA, USA), with GAPDH used as a reference control. The primer sequences are listed in Table 1.

\section{Propidium iodide staining}

After the cells were treated with $\mathrm{M} 1 \mathrm{CM}$ and Q-M1 CM, propidium iodide (PI) $(5 \mu \mathrm{g} / \mathrm{ml}$, Sigma, Santa Clara, USA) and Hoechst $33342(3 \mu \mathrm{g} / \mathrm{ml}$, Sigma, Santa Clara, USA) were added into the culture medium and incubated for $20 \mathrm{~min}$ at $37{ }^{\circ} \mathrm{C}$. After washing with $0.01 \mathrm{M}$ PBS three times, cells were fixed with 4\% PFA for $10 \mathrm{~min}$ at RT. Cells were then imaged and counted under inverted fluorescence microscope (IX71, Olympus, Tokyo, Japan).

Table 1 PCR primer sequences

\begin{tabular}{lll}
\hline Gene & Forward primer(5'-3') & Reverse primer (5'-3') \\
\hline TNFa & ATGGGCTCCCTCTCATCAGT & GCTTGGTGGTTGCTACGAC \\
iNOS & TCCTCAGGCTTGGGTCTTGT & ATCCTGTGTTGTGGGCTGG \\
CD86 & AGACATGTGTAACCTGCACCAT & TACGAGCTCACTCGGGCTTA \\
CD206 & TCAACTCTTGGACTCACGGC & CATGATCTGCGACTCCGACA \\
Arg1 & CCAGTATTCACCCCGGCTAC & GTCCTGAAAGTAGCCCTGTCT \\
IL-4 & GTACCGGGAACGGTATCCAC & TGGTGTTCCTTGTGCCGTA \\
IL-10 & CGACGCTGTCATCGATTTCTC & CAGTAGATGCCGGGTGTTC \\
IL-12 & ATCATCAAACCGGACCCACC & AGGAACGCACCTTCTGGTT \\
IL-13 & GCTTCCTTGTGCAAGTGTCT & TCTGGACAGCCCAAGTCAAG \\
TGFB & CTGCTGACCCCCACTGATAC & AGCCCTGTATTCCGTCTCCT \\
GAPDH & AGTGCCAGCCTCGTCTCATA & GGTAACCAGGCGTCCGATAC \\
\hline
\end{tabular}




\section{ROS measurement}

DCFH-DA probe (Sigma, St. Louis, MO, USA) was used to detect reactive oxygen species (ROS) levels. DCFHDA $(10 \mu \mathrm{M})$ was added to 96-well plates seeded with OLs incubated for $30 \mathrm{~min}$ at $37^{\circ} \mathrm{C}$. ROS levels were then examined by a multimode microplate reader (TECAN, Infinite M200, Mannedorf, Switzerland).

\section{ATP measurement}

ATP levels were measured using the Cell Titer-Glo assay kit (Promega, Madison, USA). Equivalent Cell Titer-Glo Reagent was added into medium for $5 \mathrm{~min}$ to induce cell lysis, followed by incubating for $15 \mathrm{~min}$ to stabilize luminescent signal. Luminescence was detected by multimode microplate reader (TECAN, infinite M200, Mannedorf, Switzerland).

\section{Quantification of cells}

In our study, the method of profile counts followed by calibration with the empirical method was adopted for unbiased data $[23,24]$.The $12-\mu \mathrm{m}$ thickness of the section ensures that the plane of central canal is cut through on every slide, making random selection of the slide possible. For cells quantification, all of the positive cells in the defined area in all sections of the randomly chosen slide were counted. Then calibration of the profile numbers was made by empirical method. Cell counting was performed by an observer who was blinded to the experiment design.

For quantitative analysis of density of MBP and NF200, serial transverse cryosections (12- $\mu$ m thickness) were collected every millimeter section rostral and caudal $2000 \mu \mathrm{m}$ to the lesion site. The longitudinal sections were also performed to confirm the density of MBP. The data were presented as a percentage relative to that in sham control (100\%).

\section{Statistics}

Quantification was performed by researchers who were blinded to the group allocation. No data were excluded from the analysis, except for five rats evaluated by $\mathrm{BBB}$ scores. In the present study, data are expressed as the mean \pm standard error of the mean (SEM). Comparisons between two groups were evaluated by the Student's $t$ test [25]. For multiple comparisons with the three or four groups, we performed one-way analysis of variance (ANOVA), followed by Tukey post-hoc tests, except for $\mathrm{BBB}$ scoring and RHI assay which were further analyzed by the Bonferroni post hoc test as recommended $[26,27]$. Differences were considered statistically significant if $* p<0.05, * p<0.01$, or ${ }^{* * *} p<0.001$.

\section{Results}

Quercetin improved functional recovery after SCI

Locomotor functional recovery was evaluated using $\mathrm{BBB}$ scoring and RHI assay at 1 day before injury as well as 1 , $3,7,10,14$, and 21 days after SCI. Compared with SCI + saline control, the quercetin-treated rats showed significant higher $\mathrm{BBB}$ scores from $7 \mathrm{dpi}(n=6$, " $p<0.05$, Fig. 1a), which was consistent with the previous results [16]. Similarly, the rump height shown by RHI was significantly elevated from $7 \mathrm{dpi}$ within quercetin-treated group ( $n=6$ rats/group, $* p<0.05$, Fig. $1 \mathrm{~b}$ ).

Quercetin prevented necroptosis of OLs without affecting apoptosis and regeneration of OLs after $\mathrm{SCl}$

Given that the survival of OLs is crucial for functional recovery after SCI $[4,28]$, we then focused on death and

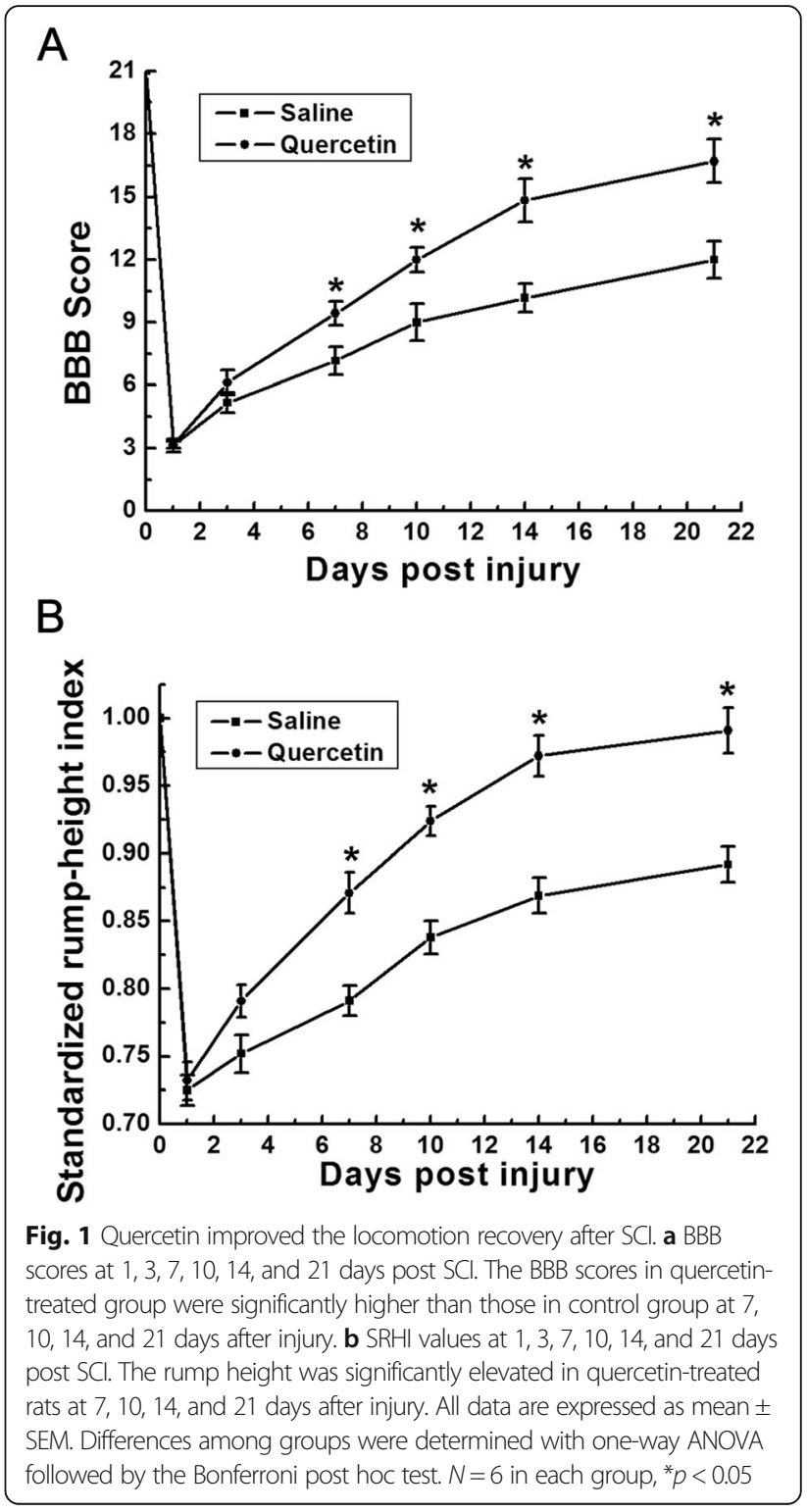


regeneration of OLs. Apart from apoptosis, RIP3/MLKLmediated necroptosis is activated after $\mathrm{SCI}$ and our previous study showed that CC1/MLKL double-positive cells appeared after SCI [9], indicating the necroptosis of OLs. To investigate the effect of quercetin on necroptosis of OLs after SCI, double staining of RIP3/CC1, MLKL/CC1, and $\mathrm{pMLKL/CC1} \mathrm{were} \mathrm{performed} \mathrm{at} 10$ days post injury. The data of double staining showed that the number of RIP3/CC1, MLKL/CC1, and pMLKL/CC1 was reduced by $59.05 \pm 3.57 \%, 56.92 \pm 3.59 \%, 43.3 \pm$ $1.07 \%$, and $71.11 \pm 3.82 \%$ respectively after quercetin treatment $(n=6, * p<0.05$, Fig. 2a-f). Moreover, quercetin improved OLs survival by about $80 \%$ shown by CC1-positive cells in our model ( $n=6, " p<0.05$, Fig. 2g, h). None of the RIP3-, MLKL-, and pMLKL-positive cells were observed in sham-operated cords (data not shown). The above results indicated that quercetin treatment significantly reduced the numbers of necroptosis of OLs after SCI.

Further, we examined that whether quercetin could improve OL preservation only or enhance both OL preservation and OL regeneration after SCI. BrdU administration scheme was shown in Fig. 3a. By co-immunostaining of PDGFR $\alpha / \mathrm{BrdU}$ and $\mathrm{CC} 1 / \mathrm{Olig} 2$, we found that quercetin had no significant effect on OL regeneration after SCI (Fig. 3b, c). In addition, by co-immunostaining of PDGFR $\alpha$ and RIP3, MLKL, or PMLKL, we found that there are none of the double-positive cells (Fig. 3d), indicating that none of the OPCs occurred necroptosis.

Moreover, we showed that just a small proportion of OLs(about 2.3\%) died through apoptosis in the first 5 days after SCI, and from 5 dpi to $10 \mathrm{dpi}$, more OLs

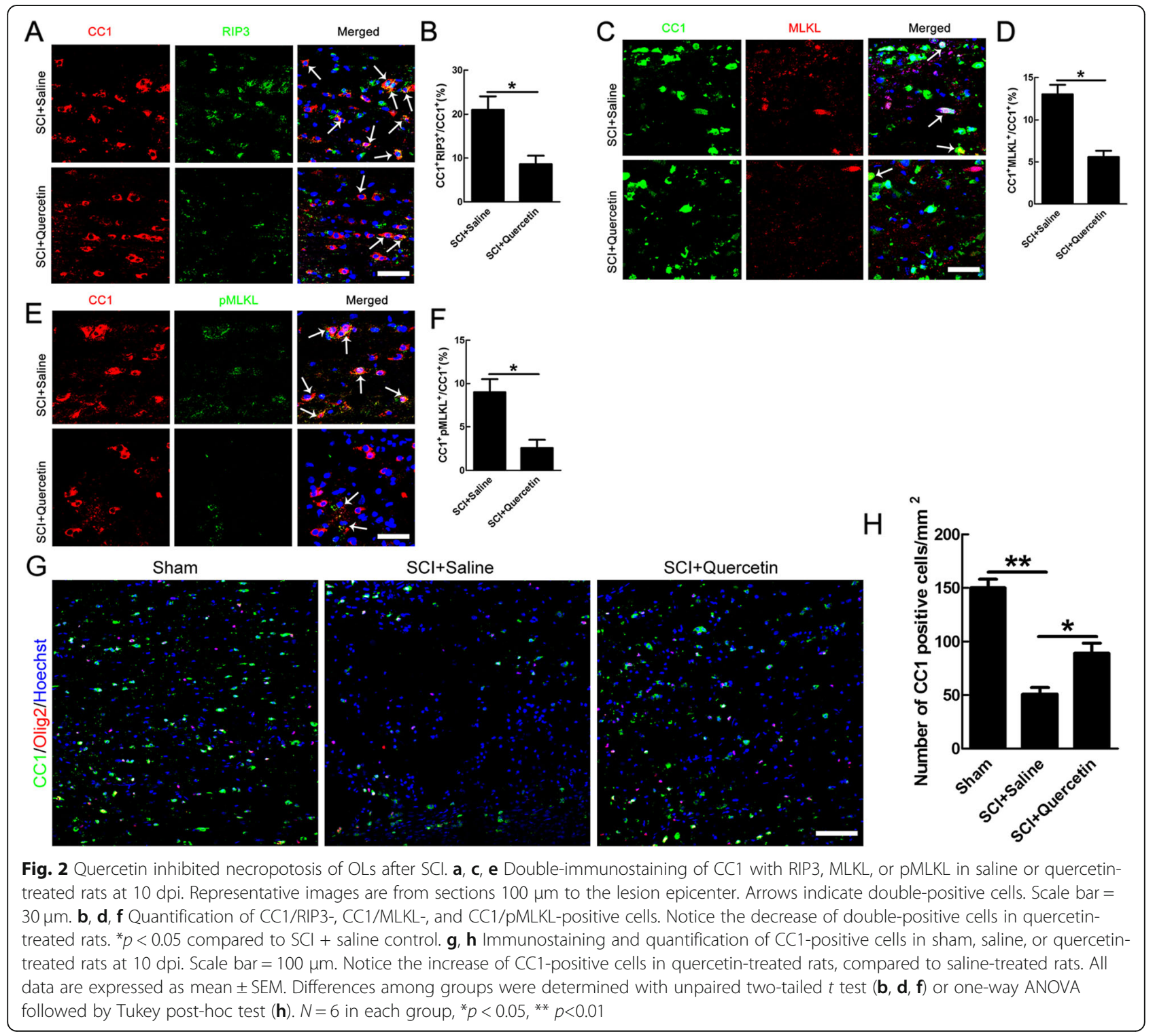



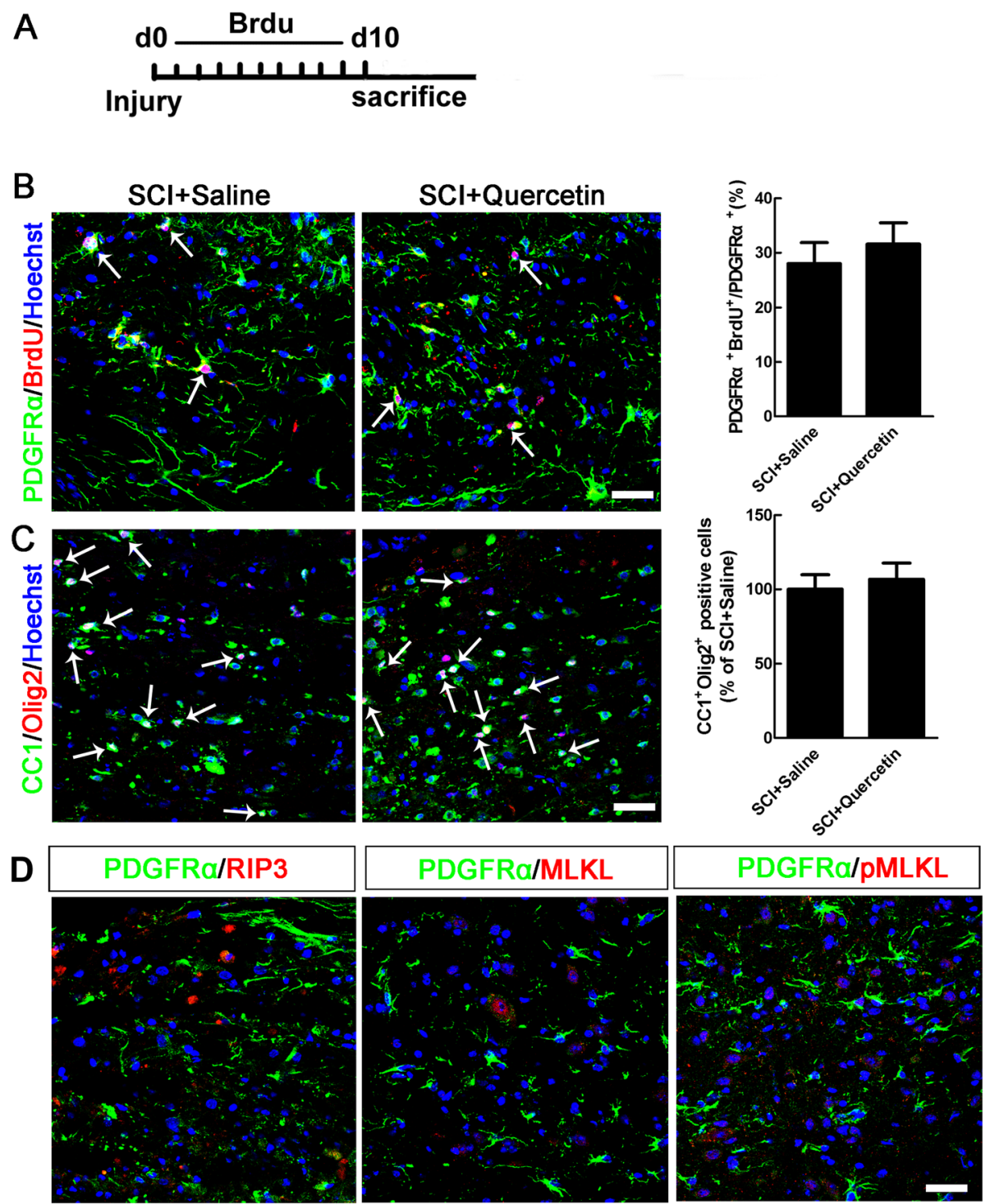

Fig. 3 Quercetin had no effect on regeneration and apoptosis of OLs after SCl. a BrdU administration scheme. b, c Co-immunostaining of PDGFRa/BrdU and CC1/Olig2, and quantification of double-positive cells. Note that no significant effect of quercetin on OL regeneration after SCl. d Double-immunostaining of PDGFRa and RIP3, MLKL, or pMLKL. Notice that none of the double-positive cells was found. Scale bars $=50 \mu \mathrm{m}$. All data are expressed as mean \pm SEM. Differences among groups were determined with unpaired two-tailed $t$ test $(\mathbf{b}, \mathbf{c})$. $N=6$ in each group

were eliminated by necroptosis (about 13\%) rather than apoptosis (about 3.1\%) (Additional file 1: Figure S1a-c), indicating that necroptosis was the main way of OLs loss in our model of SCI. In addition, no significant effect of quercetin on apoptosis of OLs was found (Additional file 1: Figure S1d-e).

\section{Quercetin reduced myelin and axonal loss after $\mathrm{SCI}$}

Given that death of OLs results in demyelination and secondary axon damage $[29,30]$, we examined the effect of quercetin on loss of myelin and axons after SCI. Spinal cords at 21 days after injury were processed for LFB staining and immunostaining to assess the myelin
loss(Fig. 4a, b). In both dorsal and lateral funiculus of the white matter at $2000 \mu \mathrm{m}$ rostral from the lesion and longitudinal sections, myelin and axonal loss was extensive in the SCI + saline control, whereas quercetin treatment significantly attenuated MBP-positive myelin and NF200-positive axonal loss $\left(n=6\right.$; ${ }^{* * *} p<0.01, " p<0.05$, Fig. 4a-d). Demyelination was also observed in the same region of white matter by electron microscopic analysis, and the data showed that SCI induced decompaction of myelin sheaths with a decreased g-ratio, an increased large-diameter axons, and a decreased axonal number, whereas quercetin treatment attenuated these effects $(n=$ 6; " $p<0.05$, Fig. 4e-h). These data indicated that 

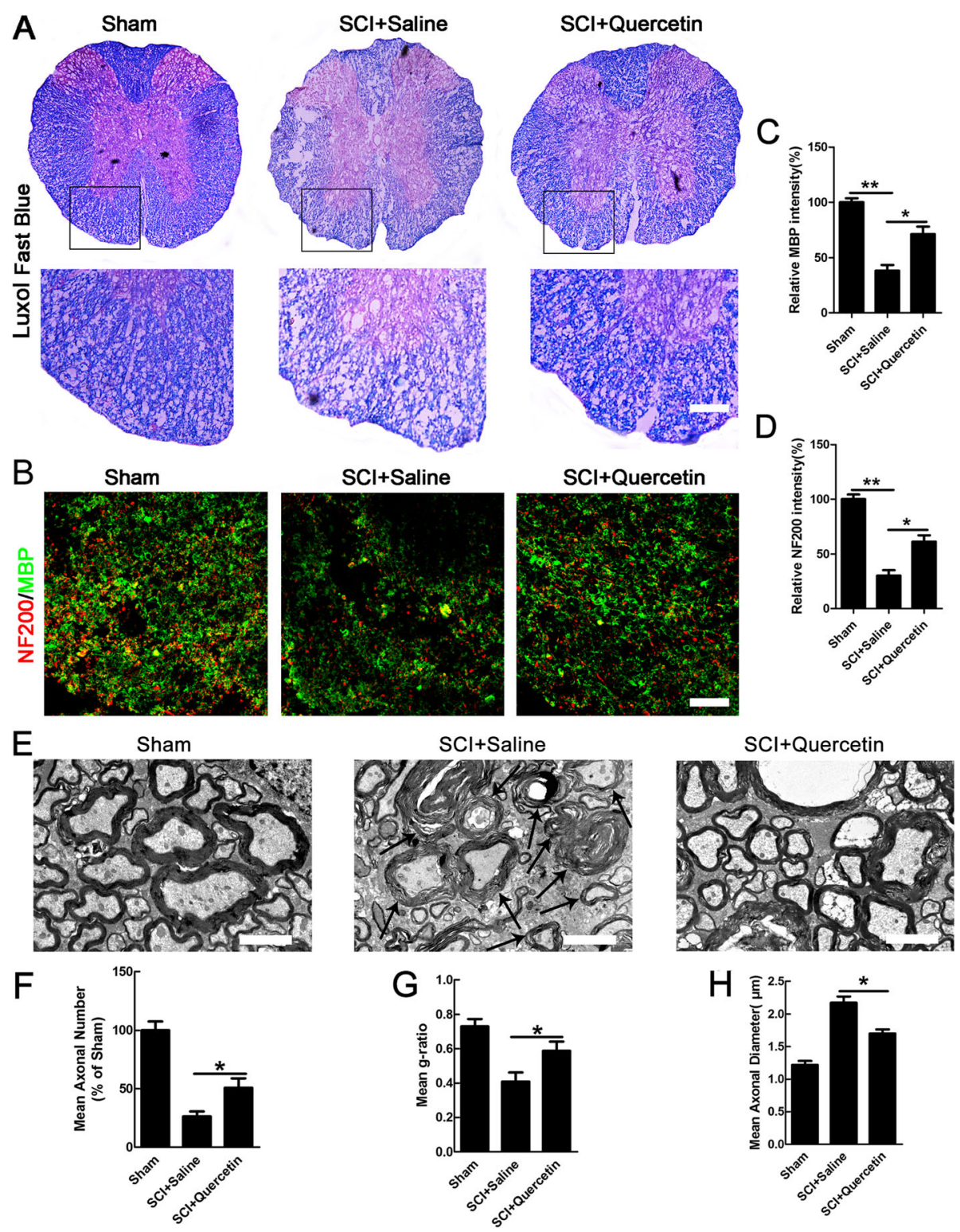

Fig. 4 Quercetin reduced myelin and axonal loss after SCl. a Spinal cords at 21 dpi were processed for Luxol fast blue. b Double-staining of MBP/ NF200 in transverse sections. Immunofluorescence image of MBP and NF200 in lateral and dorsal funiculus of cords in sham, saline, or quercetintreated rats at $21 \mathrm{dpi}$ after SCl. Transverse cryosections were selected $2000 \mu \mathrm{m}$ rostral to the lesion site. Scale bar $=100 \mu \mathrm{m}$. c, d Quantification of MBP and NF200 intensity. Note that quercetin treatment attenuated the reduction of MBP and NF200 in the white matter after injury. e-h Electron microscopic images and quantification of the mean axonal numbers, mean g-ratios, and mean axonal diameters in spinal cord white matter at $21 \mathrm{dpi}$. Arrows indicate degenerated myelin exhibiting onion-like appearance whose myelin lamellae were disorganized and loosened. Note that the increased number of axonal numbers, mean g-ratios, and decreased mean axonal diameters in quercetin treated rats. Scale bar $=$ $2 \mu \mathrm{m}$. All data are expressed as mean \pm SEM. Differences among groups were determined with one-way ANOVA followed by Tukey post-hoc test $(\mathbf{c}, \mathbf{d}, \mathbf{f}, \mathbf{h}) . N=6$ in each group, ${ }^{*} p<0.05 ;{ }^{* *} p<0.01$

quercetin treatment markedly reduced the extent of both myelin and axon loss after SCI.

\section{Quercetin influenced the macrophages/microglia M1-M2} polarization balance after $\mathrm{SCI}$

Considering that the ameliorated cell necroptosis needs a permissive immuno-microenvironment and quercetin has been used as an anti-inflammatory agent $[10,31]$, we then explored the effects of quercetin on macrophages/ microglia-mediated inflammation after SCI. The activation of macrophages/microglia can be divided to proinflammatory, damaging M1 phenotype, and antiinflammatory, regeneration-promoting M2 phenotype after SCI [32]. Because the anti-inflammatory effect of 
quercetin has been reported in SCI [17, 33], we speculated that quercetin could inhibit macrophages/microglia polarization to M1 phenotype. To determine the effect of quercetin on M1 polarization, we first examined the mRNA levels of TNF $\alpha$, iNOS, and CD86, the markers of M1 macrophages/microglia at 10 days after injury. Our data showed that mRNA of TNF $\alpha$, iNOS, and CD86 was decreased by $50.17 \pm 2.43 \%, 45.23 \pm 2.06 \%$, and $59.14 \pm$ $3.51 \%$ respectively in the quercetin group ( $n=6$ rats/ group, " $p<0.05$, Fig. $5 \mathrm{a}-\mathrm{c}$ ). Meanwhile, quantification in bilateral areas $200 \mu \mathrm{m}$ rostral and dorsal to lesion center, as shown in Fig. 5f, demonstrated that quercetin decreased the number of iNOS-expressing macrophages/ microglia by $59.1 \pm 2.26 \%(" p<0.05$, Fig. 5 d, e).

We further examined the effect of quercetin on M2 polarization. Interestingly, the results showed that quercetin increased the mRNA levels of Arginase1, IL-4, and CD206 by $45.09 \pm 2.14 \%, 65.89 \pm 5.34 \%$, and $61.9 \pm 3.75 \%$ respectively ( $n=6$ rats/group, $" p<0.05$, Fig. $6 \mathrm{a}-\mathrm{c}$ ). Because M2 macrophages/microglia was mainly located in the epicenter after SCI [34, 35], we analyzed the numbers of $\mathrm{M} 2$ in the inner border of GFAP-immunoreactive zone (Additional file 2). The result showed that the Arginase1-positive macrophages/microglia was increased by $46.44 \pm 4.79 \%(" p<$ 0.05 , Fig. $6 \mathrm{~d}, \mathrm{e})$. None of the iNOS- or Arginase1positive cells was observed in sham-operated cords (data not shown). Collectively, the above results showed that quercetin could inhibit macrophages/ microglia polarization to M1 phenotype, while promote M2 polarization, which may forms an environment allowing for survival of OLs.
A
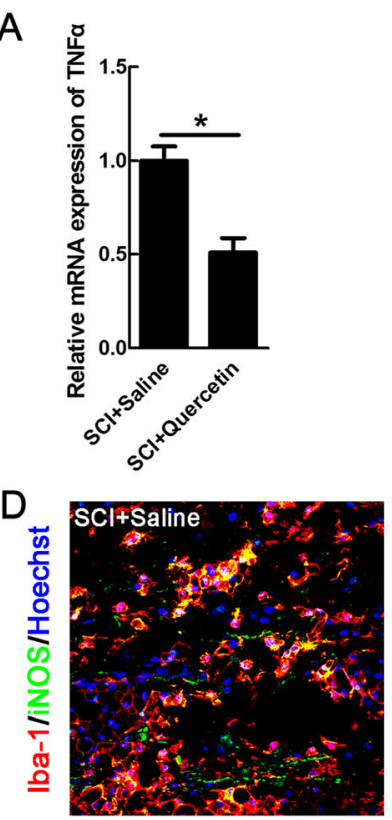

F

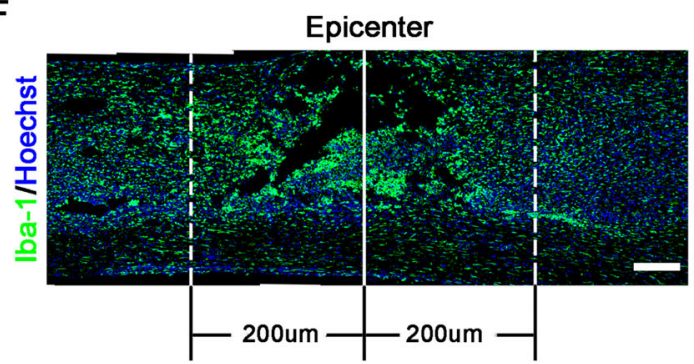

B
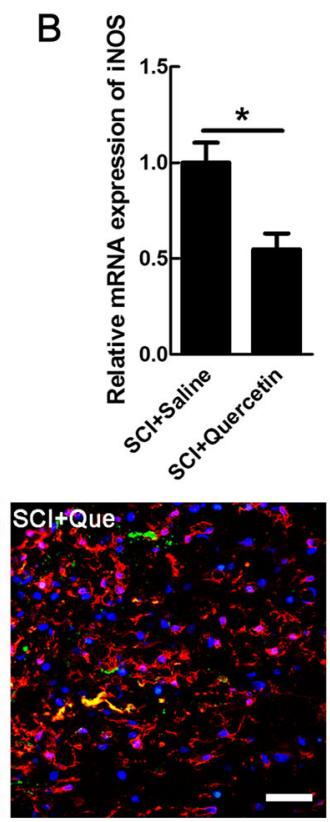

Epicenter
C

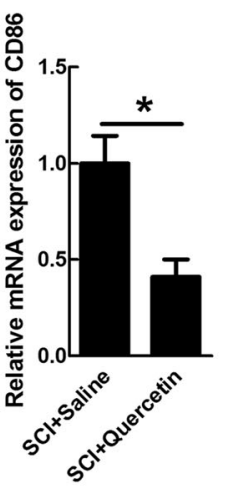

E

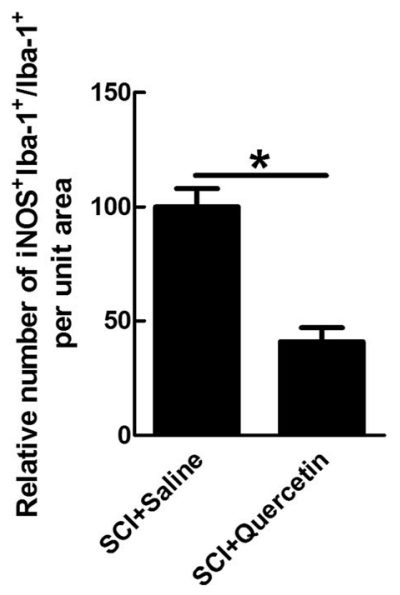

Fig. 5 Quercetin reduced the numbers of M1-type macrophages/microglia in injured spinal cord. a-c Real-time reverse-transcriptase polymerase chain reaction of M1-associated MRNA transcripts of TNFa, iNOS, and CD86 in saline or quercetin-treated rats at 10 dpi. $\mathbf{d}$ Double-staining of iNOS and Iba-1 in quercetin-treated or saline-treated rats at $10 \mathrm{dpi}$. Representative images are from sections $20 \mu \mathrm{m}$ to the lesion epicenter. Scale bar $=30 \mu \mathrm{m}$. e Quantification of iNOS-positive microglia/macrophages. Notice the decrease of iNOS-positive microglia/macrophages in quercetintreated rats. ${ }^{*} p<0.05$ compared to SCI + saline control. $\mathbf{f}$ The bilateral areas $200 \mu \mathrm{m}$ rostral and caudal to the lesion site and the lesion epicenter on which quantification was performed. Scale bar $=80 \mu \mathrm{m}$. All data are expressed as mean \pm SEM. Differences among groups were determined with unpaired two-tailed $t$ test $(\mathbf{a}-\mathbf{c}, \mathbf{e}) . N=6$ in each group, ${ }^{*} p<0.05$ 
A
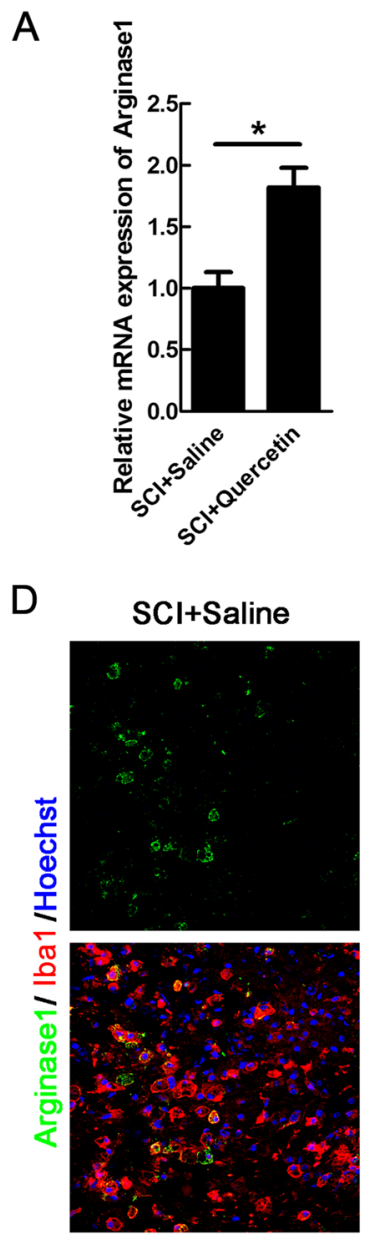

B

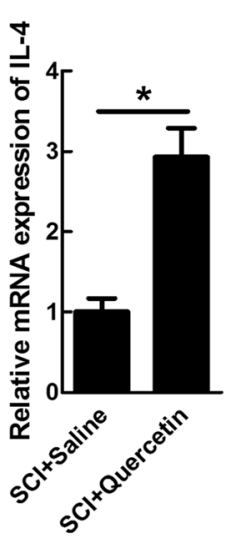

C

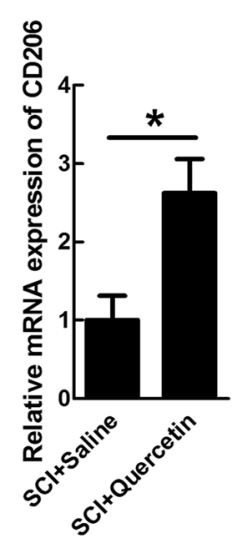

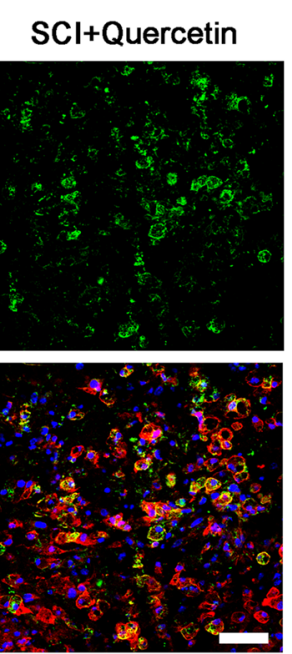

E

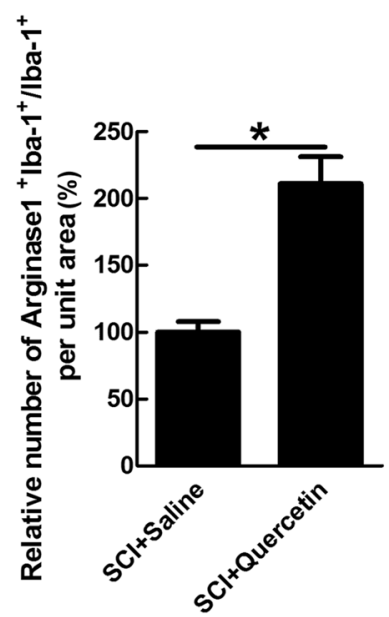

Fig. 6 Quercetin increased the numbers of M2-type macrophages/microglia after SCl. a-c Expression of M2-associated mRNA transcripts of Arginase1, IL-4, CD206 in saline, or quercetin-treated rats at 10 dpi. d Double-staining of Arginase 1 and Iba-1 in quercetin-treated or saline-treated rats at $10 \mathrm{dpi}$. Scale bar $=30 \mu \mathrm{m}$. e Quantification of the numbers of Arginase1-positive microglia/macrophages. Sections were taken from the lesion epicenter. All data are expressed as mean \pm SEM. Differences among groups were determined with unpaired two-tailed $t$ test (a-c, e). $N=6$ in each group, ${ }^{*} p<0.05$

The mechanism of effect of quercetin on M1 macrophages/microglia-induced necroptosis of OLs The pro-inflammatory response is always accompanied with cell death after SCI [36]. To explore the direct evidence that M1 macrophages/microglia contributes to necroptosis of OLs, we further cultured OLs and microglia and induced necroptosis. MBP staining was performed to identify the purity of OLs, and about $80 \%$ of the cells are MBP positive (data not shown). Quercetin-modified M1 $\mathrm{CM}$ (Q-M1 CM) was obtained by addition of quercetin to microglia when polarizing them to M1 phenotype. After treatment with conditioned medium of M1 microglia (M1 $\mathrm{CM}$ ), the ROS level of OLs increased 3.3-fold, ATP level decreased 2.4-fold, PI labeled cells increased 6.7-fold, which were the indicators of necroptosis [37, 38] $(n=3, * p<0.01$, Fig. 7a, b, g, h). The expression of RIP3, MLKL, and pMLKL was also significantly enhanced by M1 CM $(n=3$, $* * 0<0.01, " p<0.05$, Fig. $7 \mathrm{c}-\mathrm{f})$. The effects were significantly blocked by Q-M1 CM, and quercetin alone has no significant effects on necroptosis of OLs $(n=3, * p<0.05$, Fig. $7 \mathrm{a}-\mathrm{h}$ ). These data suggested that quercetin inhibited necroptosis of OLs induced by M1 macrophages/microglia.

To determine whether this reduction resulted from the suppressed polarization of M1 macrophages/microglia, mRNA levels of M1-related markers and M2related markers were detected. The results showed that quercetin decreased the mRNA levels of TNF $\alpha$, IL-12, and IL-1 $\beta$ in M1 microglia, while increased mRNA levels of IL-4, IL-10, and TGF- $\beta(n=3, * * * p<0.001, * * p<0.01$, ${ }^{*} p<0.05$, Fig. $8 \mathrm{a}, \mathrm{b}$ ). The data provides the direct evidence that quercetin prevented macrophages/microglia polarized to M1 phenotype.

Further, we explored the possible mechanism of the above effect by examining the STAT1 and NF- $\mathrm{kB}$ signaling pathway, which was reported to be involved in transcriptional regulation of M1polarization of macrophages/ 

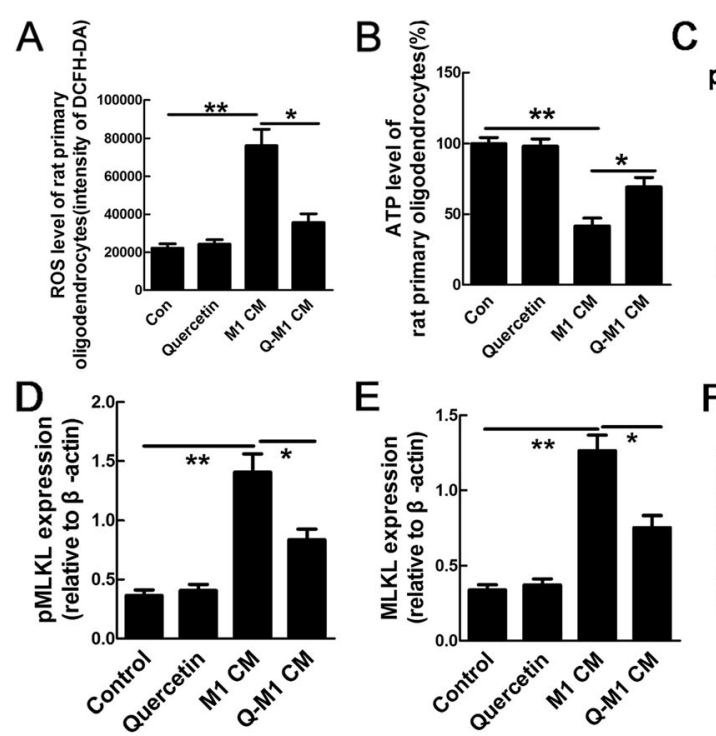

$\mathrm{E}$
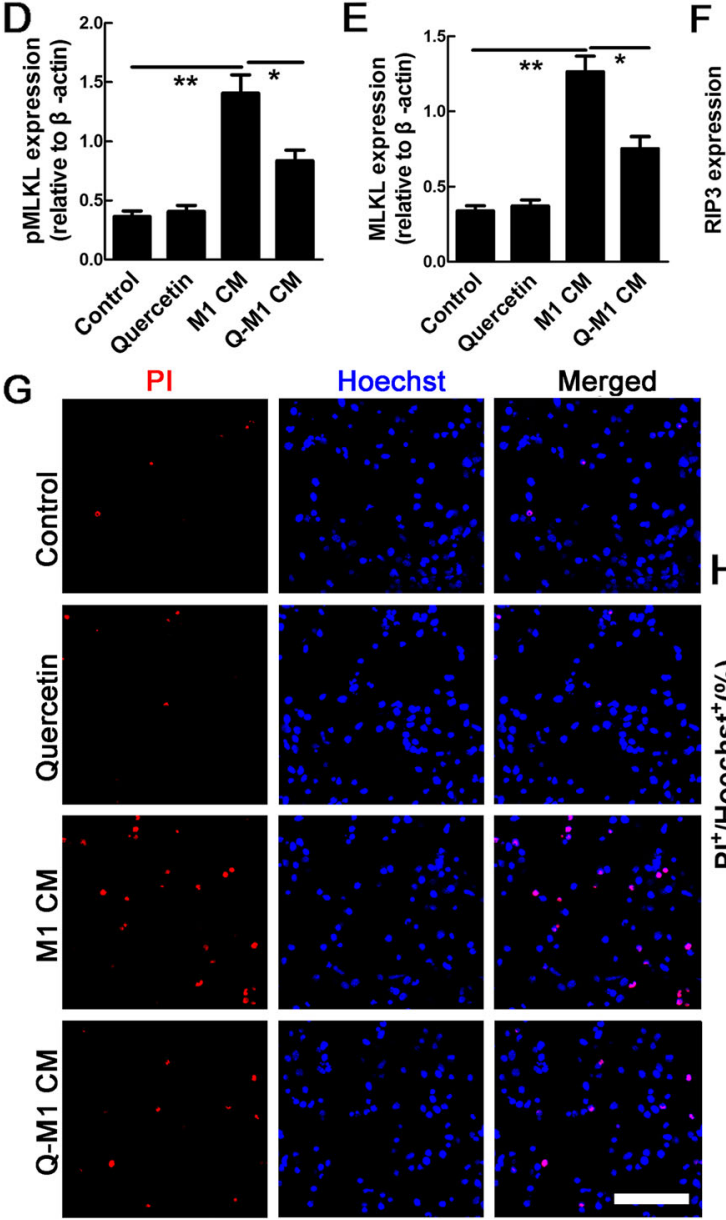

$F$
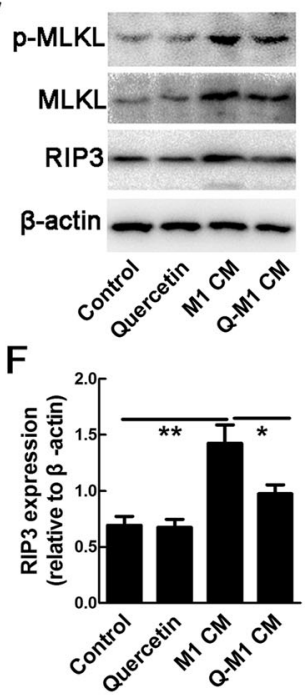

$\mathrm{H}$
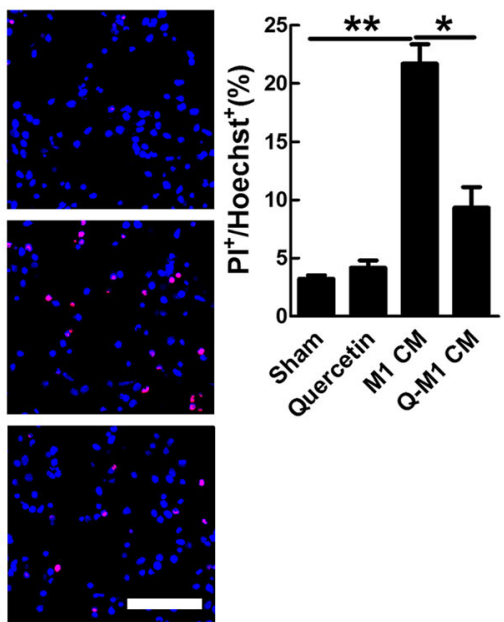

Fig. 7 Quercetin inhibited M1 macrophages/microglia induced-necroptosis of OLs. a, b Quantification of intracellular ROS and ATP levels in OLs, or cells treated by quercetin alone, conditioned medium (CM) from M1 microglia (M1 CM) and quercetin-treated M1 microglia (Q-M1 CM). Notice that quercetin significantly attenuated the effects of M1 CM on necroptosis of OLs. c-f Quantification of the expression levels of RIP3, MLKL, and pMLKL in control cells, quercetin alone-treated, M1 CM-treated, or Q-M1 CM-treated cells. $\beta$-actin was used as a loading control. Notice that quercetin significantly decreased the expression of necroptotic markers induced by M1 CM. g, h PI staining and quantification of PI-positive cells in control cells, or cells treated by quercetin alone, M1 CM, and Q-M1 CM. Notice that quercetin significantly decreased the number of PI-labeled cells induced by M1 CM. Scale bar $=50 \mu \mathrm{m}$. Results are mean \pm SEM of three independent experiments. Differences among groups were determined with one-way ANOVA followed by Tukey post-hoc test $(\mathbf{a}, \mathbf{b}, \mathbf{d}-\mathbf{f}, \mathbf{h}) .{ }^{*} p<0.05$; ${ }^{* *} p<0.01$

microglia. In vitro, the expression of iNOS, pSTAT1, NF$\kappa \mathrm{B}$, and $\mathrm{p}-\mathrm{NF}-\mathrm{\kappa B}$ was decreased by $56.94 \pm 2.49 \%, 58.31 \pm$ $5.86 \%, 56.6 \pm 3.46 \%$, and $53.33 \pm 9.58 \%$ respectively when quercetin was added to M1 microglia $(n=3, * * p<0.01$, $* p<0.05$, Fig. 8c, d). In vivo, SCI dramatically induced the expression of iNOS, pSTAT1, NF-kB, and p-NF$\kappa B$, and quercetin treatment significantly reduced their expressions by $42.86 \pm 4.01 \%, \quad 51.28 \pm 2.41 \%$, $30.43 \pm 3.46 \%$, and $57.37 \pm 6.16 \%$ respectively $(n=3$, $* p<0.01, " p<0.05$, Fig. 8e, f).

\section{Discussion}

In this study, we demonstrated that quercetin treatment improved functional recovery after SCI. Quercetin also 
A

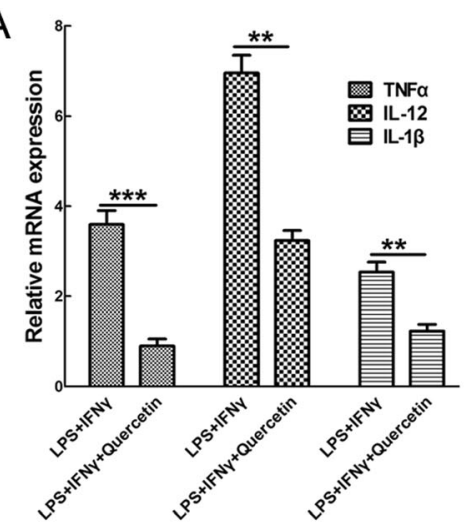

B

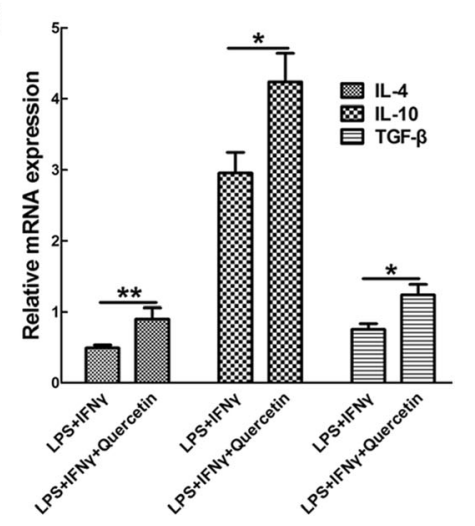

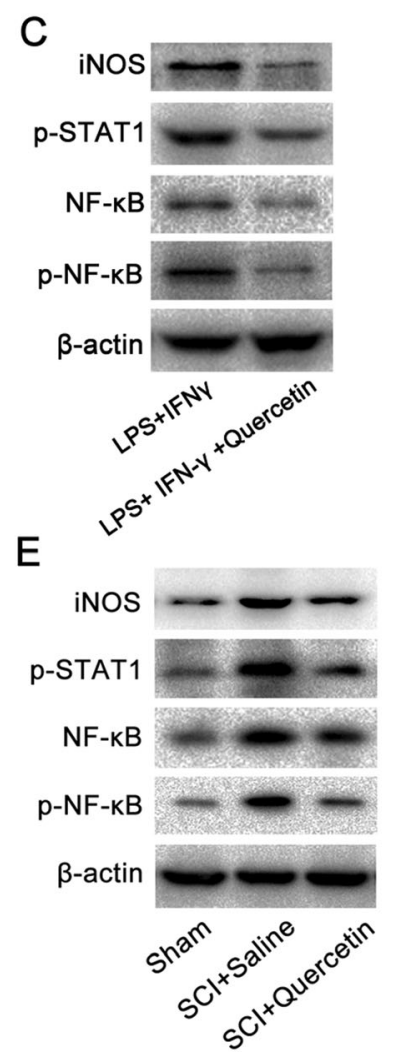

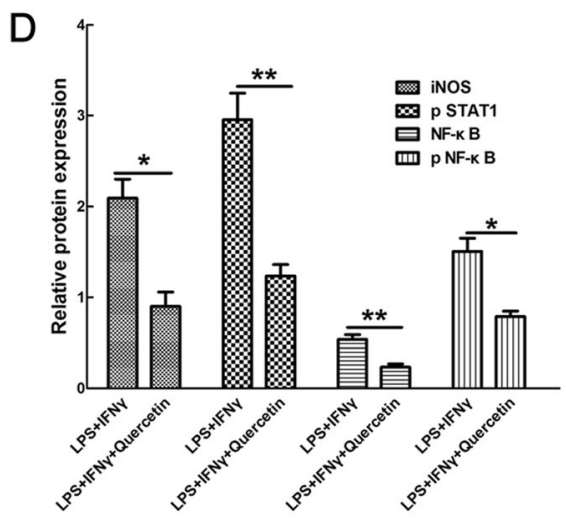

$\mathrm{F}$

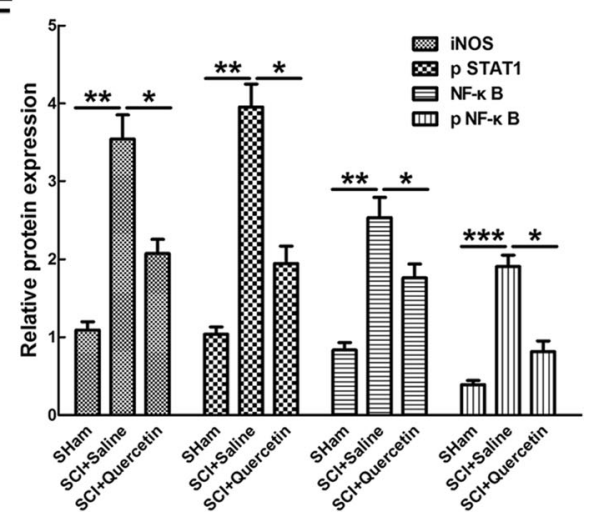

Fig. 8 Effects of quercetin on M1 polarization of microglia and expression of pSTAT1, NF-KB, and pNF-KB. $\mathbf{a}$, $\mathbf{b}$ The effect of quercetin on mRNA levels of $\mathrm{M} 1$ related TNFa, IL-12, IL-1 $\beta$, and M2-related IL-4, IL-10, and TGF- $\beta$ in M1 microglia. Note that quercetin significantly decreased mRNA levels of TNFa, IL-12, and IL-1 $\beta$, while increased mRNA levels of IL-4, IL-10, and TGF- $\beta$. Data were expressed as mean \pm SEM of three independent experiments. $\mathbf{c}$ Expression of iNOS, pSTAT1, NF-KB, and pNF-KB in microglia treated by LPS + IFN- $\gamma$ in the presence or absence of quercetin. $\mathbf{d}$ Quantification of protein levels. $\beta$-actin was used as a loading control. Data were expressed as mean \pm SEM of three independent experiments. $\mathbf{e}$ Expression of iNOS, pSTAT1, NF-kB, and pNF-KB in sham, saline, or quercetin-treated rats at $10 \mathrm{dpi}$ after SCl. $\mathbf{f}$ Quantification of protein levels. Data were expressed as mean \pm SEM of 6 rats. Differences among groups were determined with unpaired two-tailed $t$ test (a, $\mathbf{b}$, d) or one-way ANOVA followed by Tukey post-hoc test (f). ${ }^{*} p<0.05$; ${ }^{* *} p<0.01$; ${ }^{* * *} p<0.001$

attenuated RIP3/MLKL-mediated necroptosis of OLs after injury, while suppressed myelin and axon loss in the white matter after SCI. We then explored the reason and mechanism of increased number of rescued OLs after SCI, and we found that quercetin alleviated macrophages/microglia polarized to M1 phenotype, while promoted M2 polarization after SCI. The new balance of M1-M2 polarization of macrophages/microglia may form a permissive environment allowing for OL survival. Our in vitro study provided direct evidence that quercetin prevented necroptosis of OLs induced by M1 macrophages/microglia. 
As numerous intact demyelinated axons are observed after SCI [39], there is an urgent need to rescue myelin sheath. It was reported that $\mathrm{p} 75^{\mathrm{NTR}}$-mediated apoptosis of OLs can be induced by proNGF produced in activated macrophages/microglia after SCI [40, 41]. However, this acute apoptosis of OLs could not explain the chronic, delayed demyelination after SCI [42], suggesting that other types of OLs death accounted for the delayed demyelination. In our previous studies, we demonstrated that necroptosis is a chronic process and necroptosis can occur in OLs after SCI $[9,10]$; we thus focused on the effects of quercetin on necroptosis of OLs in the present study. We found that quercetin inhibited necroptosis of OLs and reduced myelin and axonal loss. Further, we examined that whether quercetin could improve OL preservation only or enhance both OL preservation and $\mathrm{OL}$ regeneration after SCI. In the previous study, quercetin has been found to promote proliferation and differentiation of OPCs after oxygen/glucose deprivation-induced injury in vitro [43], which is a direct effect of quercetin on OPCs. However, in this study, no significant difference of OL regeneration between control and quercetin-treated rats was found, and we speculated that the microenvironment is one of the possible reasons for the different results between in vitro and in vivo. Moreover, no significant effect of quercetin on apoptosis of OLs was observed. Thus, in the present study, we concluded that quercetin inhibited necroptosis of OLs without influencing regeneration and apoptosis of OLs.

Spinal cord injury can activate quiescent microglia polarized to M1/M2 phenotypes as well as the recruited macrophages. The distinct phenotype may exert their respective roles in pathological event of SCI. The inflammatory response in $\mathrm{SCI}$ is characterized by predominant and prolonged M1 macrophages/microglia polarization [35], which forms a detrimental environment for OL survival. Quercetin, a compound acting as anti-oxidative and anti-inflammatory, has been shown to have neuroprotective effects partially by inhibiting inflammatory response after SCI [16]. In our study, we mainly focused on the effect of quercetin on polarization of macrophage/microglia and the data revealed that quercetin suppressed macrophages/microglia polarization to M1 phenotype, while promoted M2 polarization without affecting the total amount of macrophage/microglia.

After hemorrhagic brain injury, reduced M1polarization of microglia was reported by inhibiting TLR4 [44], and we speculated that the difference between our study and the previous research is that we combined LPS and IFN $\gamma$ to treat cells. It is also known that activation of STAT1 and NF- $\mathrm{KB}$ signaling pathways can skew macrophages/microglia toward the M1 phenotype [45]. In this study, we found that quercetin suppressed macrophages/microglia polarized to M1 phenotype through inhibition of STAT1 and NF-KB pathway, which was consistent with the previous results from BV2 cell lines [46]. Nevertheless, the specific mechanism for inhibition of M1 polarization by quercetin requires further study.

Time window for administration of agents is pivotal to therapeutic effect [47]. Although the previous study showed that neuroprotection can be found when quercetin was administered twice daily for a period of either 3 or 10 days [19], we selected the latter because the M1 macrophages/microglia-mediated inflammatory response is a chronic and long-lasting period [35]. In addition, in our preliminary experiment, quercetin alone was administrated to normal rats without SCI, and no effect was found.

Since the functions of microglia and macrophages are similar but not exactly the same $[48,49]$, a major challenge after SCI is to distinguish microglia and recruited macrophages to determine their phenotype and function. In the present study, we investigated the effects of quercetin on polarization of microglia/macrophages, and one general limitation of our research is that these two distinct cells had not been distinguished. It was reported that application of genetic reporter mice and flow cytometry are the commonly used method to distinguish host microglia and recruited macrophages after injury [50-52]. In the present study, we applied SpragueDawley rats instead of genetic mice. Data of cytometry

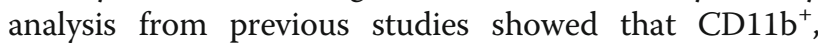
$\mathrm{CD} 45^{\text {low }}$, $\mathrm{Ly}_{6 \mathrm{G}}{ }^{-}$, and $\mathrm{Ly} 6 \mathrm{C}^{-}$represent the vast majority (>95\%) of microglia after SCI [52, 53]. Therefore, we tried our best to differentiate the host microglia and recruited macrophages by flow cytometry, but we failed to get data due to our technical restrictions. However, this important issue is worthy to be studied in future.

\section{Conclusion}

This study demonstrated that quercetin treatment alleviated necroptosis of OLs at least in part by inhibiting M1 macrophages/microglia polarization after SCI. Its beneficial effects can be potentially useful as a therapeutic agent for clinical SCI.

\section{Supplementary information}

Supplementary information accompanies this paper at https://doi.org/10. 1186/s12974-019-1613-2.

Additional file 1: Figure S1. The proportion of apoptotic and necroptotic OLs and the effect of quercetin on apoptosis of OLs. a The co-staining of Cleaved Caspase-3/MLKL/CC1 at different time points after $\mathrm{SCl}$. Scale bar $=50 \mu \mathrm{m}$. b-c Quantification of the proportion of apoptotic and necroptotic OLs at different time points after SCl. d-e Quantification and immunostaining of Cleaved Caspase-3 and CC1 in quercetin treated or $\mathrm{SCl}+$ saline control rats at $10 \mathrm{dpi}$. Note that no significant effect of quercetin on apoptosis of OLs. Scale bar $=30 \mu \mathrm{m}$. All data are expressed 
as mean \pm SEM. Differences among groups were determined with unpaired two-tailed t test (e). $N=6$ in each group.

Additional file 2: Figure S2. The area that the quantification of Arginase1-positive macrophages/microglia was performed.

\section{Abbreviations}

BBB: Basso-Beattie-Bresnahan; GFAP: Glial fibrillary acidic protein; IL: Interleukin; LFB: Luxol fast blue; MLKL: Mixed lineage kinase domain-like protein; OLs: Oligodendrocytes; OPCs: Oligodendrocyte precursor cells; PI: Propidium iodide; PVDF: Polyvinylidene difluoride; GRT-PCR: Quantitative reverse transcription polymerase chain reaction; RHI: Rump-height Index; RIP3: Receptor-interacting serine-threonine kinase 3; RIPA: Radioimmunoprecipitation assay; ROS: Reactive oxygen species; SCl: Spinal cord injury; TLR: Toll-like receptor

\section{Acknowledgments}

We thank the support from Translational Medicine Center and Department of Spine Surgeny, Hong Hui Hospital, Xi'an Jiaotong University. All experiments were conducted in compliance with the ARRIVE guidelines.

\section{Authors' contributions}

$\mathrm{HF}$ and HBT wrote the paper and performed the most of experiments. LQS, $S C L, D G H, X C, Z C, M Y$, and $X H Y$ contributed morphological study and analyzed the data. $\mathrm{HY}$ and $\mathrm{DJH}$ designed the research and revised the manuscript. All authors read and approved the final manuscript.

\section{Funding}

This work was supported by grants from the National Natural Science Foundation of China (NSFC, Grant code: 81701204) and Project funded by China Postdoctoral Science Foundation (Grant code: 2018 M643703) to Hong Fan, NSFC (Grant code: 81830077, 81472098) to Ding-Jun Hao, NSFC (Grant code: 81571208) to Hao Yang, NSFC (Grant code: 81802690) to Shi-Chang Liu, and Research Project of Xi'an Health Commission (Grant code: J201902019) to Hai-Bin Tang.

\section{Availability of data and materials}

All data generated or analyzed in this study are included in this published article and its additional files.

\section{Ethics approval and consent to participate}

All protocols were approved by the Animal Care and Use Committee of Xi'an Jiaotong University, and conformed to the Guide for the Care and Use of Laboratory Animals by the National Institutes of Health.

\section{Consent for publication}

Not applicable.

\section{Competing interests}

The authors declare that they have no competing interests.

\section{Author details}

'Shaanxi Spine Medicine Research Center, Translational Medicine Center, Department of Spine Surgery, Hong Hui Hospital, Xi'an Jiaotong University, 555 You Yi Dong Road, Xi'an 710054, Shaanxi, China. Institute of Neurosciences, Fourth Military Medical University, Xi'an 710032, Shaanxi, China. ${ }^{3}$ Department of Laboratory Medicine, Xi'an Central Hospital, Xi'an Jiaotong University, 161 Xi Wu Road, Xi'an 710003, Shaanxi, China. ${ }^{4}$ Department of Bone Microsurgery, Hong Hui Hospital, Xi'an Jiaotong University, 555 You Yi Dong Road, Xi'an 710054, Shaanxi, China.

\section{Received: 3 April 2019 Accepted: 9 October 2019}

\section{Published online: 07 November 2019}

\section{References}

1. Rong H, Zhao Z, Feng J, Lei $Y$, Wu H, Sun R, Zhang Z, Hou B, Zhang W, Sun $Y$, et al. The effects of dexmedetomidine pretreatment on the pro- and antiinflammation systems after spinal cord injury in rats. Brain Behav Immun. 2017;64:195-207.
2. Profyris C, Cheema SS, Zang D, Azari MF, Boyle K, Petratos S. Degenerative and regenerative mechanisms governing spinal cord injury. Neurobiol Dis. 2004;15:415-36.

3. Beattie MS, Hermann GE, Rogers RC, Bresnahan JC. Cell death in models of spinal cord injury. Prog Brain Res. 2002;137:37-47.

4. Papastefanaki F, Matsas R. From demyelination to remyelination: the road toward therapies for spinal cord injury. Glia. 2015;63:1101-25.

5. Beattie MS, Li Q, Bresnahan JC. Cell death and plasticity after experimental spinal cord injury. Prog Brain Res. 2000;128:9-21.

6. Rathnasamy $G$, Ling EA, Kaur C. Iron and iron regulatory proteins in amoeboid microglial cells are linked to oligodendrocyte death in hypoxic neonatal rat periventricular white matter through production of proinflammatory cytokines and reactive oxygen/nitrogen species. J Neurosci. 2011;31:17982-95.

7. Park E, Velumian AA, Fehlings MG. The role of excitotoxicity in secondary mechanisms of spinal cord injury: a review with an emphasis on the implications for white matter degeneration. J Neurotrauma. 2004;21:754-74.

8. Ito Y, Ofengeim D, Najafov A, Das S, Saberi S, Li Y, Hitomi J, Zhu H, Chen H, Mayo L, et al. RIPK1 mediates axonal degeneration by promoting inflammation and necroptosis in ALS. Science. 2016;353:603-8.

9. Fan $H$, Tang HB, Kang J, Shan L, Song H, Zhu K, Wang J, Ju G, Wang YZ. Involvement of endoplasmic reticulum stress in the necroptosis of microglia/ macrophages after spinal cord injury. Neuroscience. 2015;311:362-73.

10. Fan H, Zhang K, Shan L, Kuang F, Chen K, Zhu K, Ma H, Ju G, Wang YZ. Reactive astrocytes undergo M1 microglia/macrohpages-induced necroptosis in spinal cord injury. Mol Neurodegener. 2016;11:14.

11. Kroner A, Greenhalgh AD, Zarruk JG, Passos Dos Santos R, Gaestel M, David S. TNF and increased intracellular iron alter macrophage polarization to a detrimental M1 phenotype in the injured spinal cord. Neuron. 2014;83: 1098-116.

12. Boots AW, Haenen GR, Bast A. Health effects of quercetin: from antioxidant to nutraceutical. Eur J Pharmacol. 2008;585:325-37.

13. Abarikwu SO, Pant AB, Farombi EO. Dietary antioxidant, quercetin, protects sertoli-germ cell coculture from atrazine-induced oxidative damage. J Biochem Mol Toxicol. 2012;26:477-85.

14. Hogan S, Canning C, Sun S, Sun X, Zhou K. Effects of grape pomace antioxidant extract on oxidative stress and inflammation in diet induced obese mice. J Agric Food Chem. 2010;58:11250-6.

15. Wang Y, Li W, Wang M, Lin C, Li G, Zhou X, Luo J, Jin D. Quercetin reduces neural tissue damage and promotes astrocyte activation after spinal cord injury in rats. J Cell Biochem. 2018;119:2298-306.

16. Jiang W, Huang Y, Han N, He F, Li M, Bian Z, Liu J, Sun T, Zhu L. Quercetin suppresses NLRP3 inflammasome activation and attenuates histopathology in a rat model of spinal cord injury. Spinal Cord. 2016;54:592-6.

17. Zhang Y, Yi B, Ma J, Zhang L, Zhang H, Yang Y, Dai Y. Quercetin promotes neuronal and behavioral recovery by suppressing inflammatory response and apoptosis in a rat model of intracerebral hemorrhage. Neurochem Res. 2015;40:195-203.

18. Schultke E, Kendall E, Kamencic H, Ghong Z, Griebel RW, Juurlink BH. Quercetin promotes functional recovery following acute spinal cord injury. J Neurotrauma. 2003:20:583-91.

19. Schultke E, Kamencic H, Skihar VM, Griebel R, Juurlink B. Quercetin in an animal model of spinal cord compression injury: correlation of treatment duration with recovery of motor function. Spinal Cord. 2010;48:112-7.

20. Fan H, Chen K, Duan L, Wang YZ, Ju G. Beneficial effects of early hemostasis on spinal cord injury in the rat. Spinal Cord. 2016;54:1058.

21. Basso DM, Beattie MS, Bresnahan JC. A sensitive and reliable locomotor rating scale for open field testing in rats. J Neurotrauma. 1995;12:1-21.

22. Sedy J, Urdzikova L, Jendelova P, Sykova E. Methods for behavioral testing of spinal cord injured rats. Neurosci Biobehav Rev. 2008;32:550-80.

23. Coggeshall RE, Lekan HA. Methods for determining numbers of cells and synapses: a case for more uniform standards of review. J Comp Neurol. 1996;364:6-15.

24. Coggeshall RE, La Forte R, Klein CM. Calibration of methods for determining numbers of dorsal root ganglion cells. J Neurosci Methods. 1990;35:187-94.

25. Hazra A, Gogtay N. Biostatistics series module 3: comparing groups: numerical variables. Indian J Dermatol. 2016;61:251-60.

26. Sedgwick P. One way analysis of variance: post hoc testing: smoking in pregnancy. BMJ. 2014;349:97067.

27. Scheff SW, Saucier DA, Cain ME. A statistical method for analyzing rating scale data: the BBB locomotor score. J Neurotrauma. 2002;19:1251-60. 
28. Gaudet AD, Fonken LK. Glial cells shape pathology and repair after spinal cord injury. Neurotherapeutics. 2018;15:554-77.

29. Wang X, Xu JM, Wang YP, Yang L, Li ZJ. Protective effects of BMP-7 against tumor necrosis factor alpha-induced oligodendrocyte apoptosis. Int J Dev Neurosci. 2016;53:10-7

30. Pomeshchik $Y$, Kidin I, Korhonen $P$, Savchenko $E_{1}$ Jaronen $M$, Lehtonen $S$, Wojciechowski S, Kanninen K, Koistinaho J, Malm T. Interleukin-33 treatment reduces secondary injury and improves functional recovery after contusion spinal cord injury. Brain Behav Immun. 2015;44:68-81.

31. Li Y, Yao J, Han C, Yang J, Chaudhry MT, Wang S, Liu H, Yin Y. Quercetin, Inflammation and Immunity. Nutrients. 2016;8:167.

32. Xu F, Huang J, He Z, Chen J, Tang X, Song Z, Guo Q, Huang C. Microglial polarization dynamics in dorsal spinal cord in the early stages following chronic sciatic nerve damage. Neurosci Lett. 2016;617:6-13.

33. Song Y, Liu J, Zhang F, Zhang J, Shi T, Zeng Z. Antioxidant effect of quercetin against acute spinal cord injury in rats and its correlation with the p38MAPK/iNOS signaling pathway. Life Sci. 2013;92:1215-21.

34. Ma SF, Chen YJ, Zhang JX, Shen L, Wang R, Zhou JS, Hu JG, Lu HZ. Adoptive transfer of $\mathrm{M} 2$ macrophages promotes locomotor recovery in adult rats after spinal cord injury. Brain Behav Immun. 2015;45:157-70.

35. Gensel JC, Zhang B. Macrophage activation and its role in repair and pathology after spinal cord injury. Brain Res. 2015;1619:1-11.

36. Moon YJ, Lee JY, Oh MS, Pak YK, Park KS, Oh TH, Yune TY. Inhibition of inflammation and oxidative stress by Angelica dahuricae radix extract decreases apoptotic cell death and improves functional recovery after spinal cord injury. J Neurosci Res. 2012;90:243-56.

37. Degterev A, Huang Z, Boyce M, Li Y, Jagtap P, Mizushima N, Cuny GD, Mitchison TJ, Moskowitz MA, Yuan J. Chemical inhibitor of nonapoptotic cell death with therapeutic potential for ischemic brain injury. Nat Chem Biol. 2005;1:112-9.

38. Yang Z, Wang Y, Zhang Y, He X, Zhong CQ, Ni H, Chen X, Liang Y, Wu J, Zhao $S$, et al. RIP3 targets pyruvate dehydrogenase complex to increase aerobic respiration in TNF-induced necroptosis. Nat Cell Biol. 2018;20:18697.

39. McDonald JW, Belegu V. Demyelination and remyelination after spinal cord injury. J Neurotrauma. 2006;23:345-59.

40. Yune TY, Lee JY, Jung GY, Kim SJ, Jiang MH, Kim YC, Oh YJ, Markelonis GJ, Oh TH. Minocycline alleviates death of oligodendrocytes by inhibiting pronerve growth factor production in microglia after spinal cord injury. J Neurosci. 2007;27:7751-61.

41. Lee JY, Kang SR, Yune TY. Fluoxetine prevents oligodendrocyte cell death by inhibiting microglia activation after spinal cord injury. J Neurotrauma. 2015;32:633-44

42. Blight AR. Delayed demyelination and macrophage invasion: a candidate for secondary cell damage in spinal cord injury. Cent Nerv Syst Trauma. 1985;2: 299-315.

43. Wu X, Qu X, Zhang Q, Dong F, Yu H, Yan C, Qi D, Wang M, Liu X, Yao R. Quercetin promotes proliferation and differentiation of oligodendrocyte precursor cells after oxygen/glucose deprivation-induced injury. Cell Mol Neurobiol. 2014;34:463-71.

44. Lan X, Han X, Li Q, Li Q, Gao Y, Cheng T, Wan J, Zhu W, Wang J. Pinocembrin protects hemorrhagic brain primarily by inhibiting toll-like receptor 4 and reducing M1 phenotype microglia. Brain Behav Immun. 2017;61:326-39.

45. Wang $\mathrm{N}$, Liang $\mathrm{H}$, Zen K. Molecular mechanisms that influence the macrophage m1-m2 polarization balance. Front Immunol. 2014;5:614.

46. Chen JC, Ho FM, Pei-Dawn Lee C, Chen CP, Jeng KC, Hsu HB, Lee ST, Wen Tung W, Lin WW. Inhibition of iNOS gene expression by quercetin is mediated by the inhibition of IkappaB kinase, nuclear factor-kappa B and STAT1, and depends on heme oxygenase-1 induction in mouse BV-2 microglia. Eur J Pharmacol. 2005;521:9-20.

47. Young $\mathbf{W}$. The therapeutic window for methylprednisolone treatment of acute spinal cord injury: implications for cell injury mechanisms. Res Publ Assoc Res Nerv Ment Dis. 1993;71:191-206.

48. Zhou X, He X, Ren Y. Function of microglia and macrophages in secondary damage after spinal cord injury. Neural Regen Res. 2014;9:1787-95.

49. Davies CL, Miron VE. Distinct origins, gene expression and function of microglia and monocyte-derived macrophages in CNS myelin injury and regeneration. Clin Immunol. 2018;189:57-62.

50. Saederup N, Cardona AE, Croft K, Mizutani M, Cotleur AC, Tsou CL, Ransohoff RM, Charo IF. Selective chemokine receptor usage by central nervous system myeloid cells in CCR2-red fluorescent protein knock-in mice. PLoS One. 2010;5:e13693.

51. Fenrich KK, Weber P, Rougon G, Debarbieux F. Long- and short-term intravital imaging reveals differential spatiotemporal recruitment and function of myelomonocytic cells after spinal cord injury. J Physiol. 2013; 591:4895-902.

52. Zarruk JG, Greenhalgh AD, David S. Microglia and macrophages differ in their inflammatory profile after permanent brain ischemia. Exp Neurol. 2018; 301:120-32.

53. Greenhalgh AD, David S. Differences in the phagocytic response of microglia and peripheral macrophages after spinal cord injury and its effects on cell death. J Neurosci. 2014;34:6316-22.

\section{Publisher's Note}

Springer Nature remains neutral with regard to jurisdictional claims in published maps and institutional affiliations.
Ready to submit your research? Choose BMC and benefit from:

- fast, convenient online submission

- thorough peer review by experienced researchers in your field

- rapid publication on acceptance

- support for research data, including large and complex data types

- gold Open Access which fosters wider collaboration and increased citations

- maximum visibility for your research: over $100 \mathrm{M}$ website views per year

At BMC, research is always in progress.

Learn more biomedcentral.com/submissions 